Supporting Information

\title{
Design and Synthesis of Heteroleptic Cyclometalated Iridium(III) Complexes \\ Containing Quinoline-type Ligands that Exhibit Dual Phosphorescence
}

Sarvendra Kumar, ${ }^{a}$ Yosuke Hisamatsu, ${ }^{a}$ Yusuke Tamaki, ${ }^{b}$ Osamu Ishitani ${ }^{b}$ and Shin Aoki ${ }^{a, c *}$

${ }^{a}$ Faculty of Pharmaceutical Science, Tokyo University of Science, 2641 Yamazaki, Noda, Chiba 278-8510, Japan,

Phone no. $+81-4-7121-3670$

${ }^{b}$ Department of Chemistry, Graduate School of Science and Engineering, Tokyo Institute of Technology, 2-12-1-NE-1 O-okayama, Meguro-Ku, Tokyo 152-8550, Japan.

${ }^{\mathrm{c}}$ Imaging Frontier Cancer, Research Institute for Science and Technology, Tokyo University of Science, 2641 Yamazaki, Noda, Chiba 278-8510, Japan. 

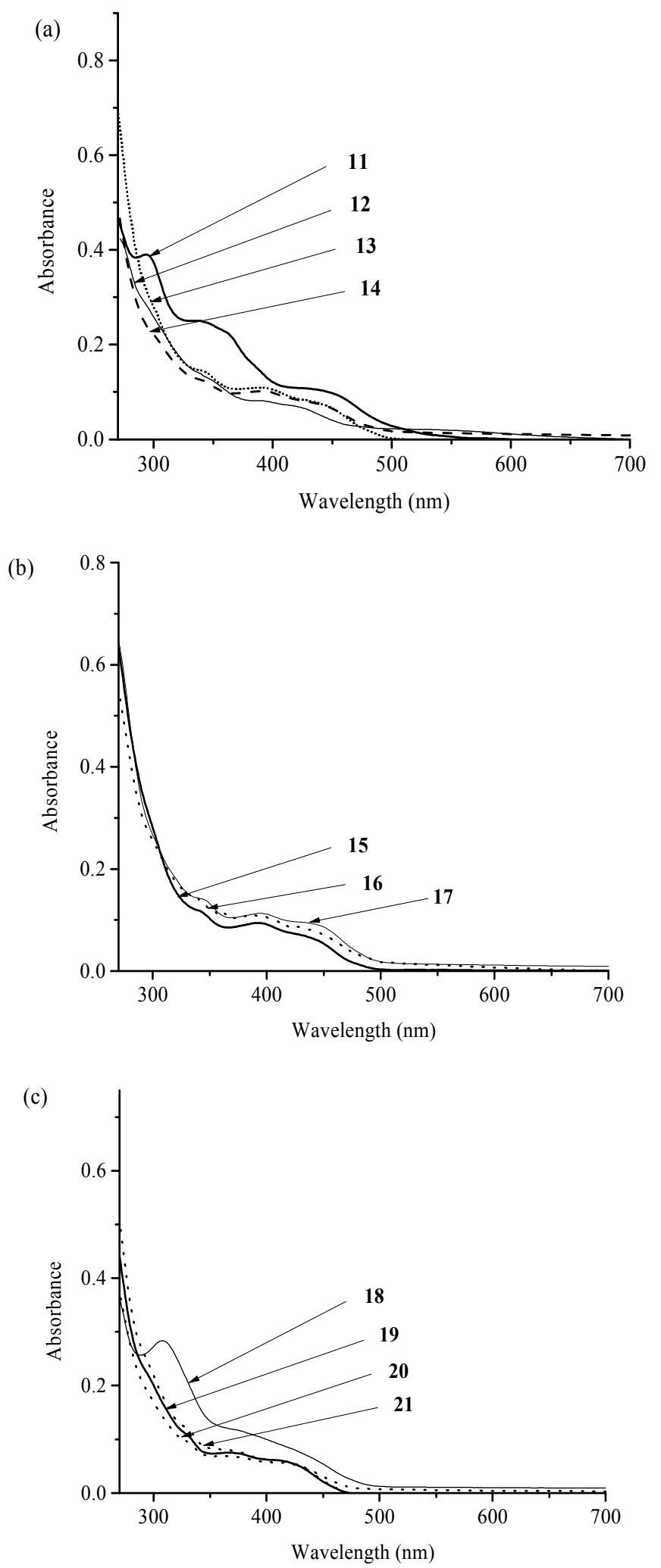

Figure S1. UV/vis spectra of (a) 11 (bold curve), 12 (plain curve), 13 (dotted curve) and 14 (dashed curve), (b) 15 (bold curve), 16 (dotted curve) and 17 (plain curve), and (c) 18 (plain curve), 19 (bold curve), 20 (dashed curve) and 21 (dotted curve) in DMSO at 298K ([Ir complex $]=10 \mu \mathrm{M})$. 

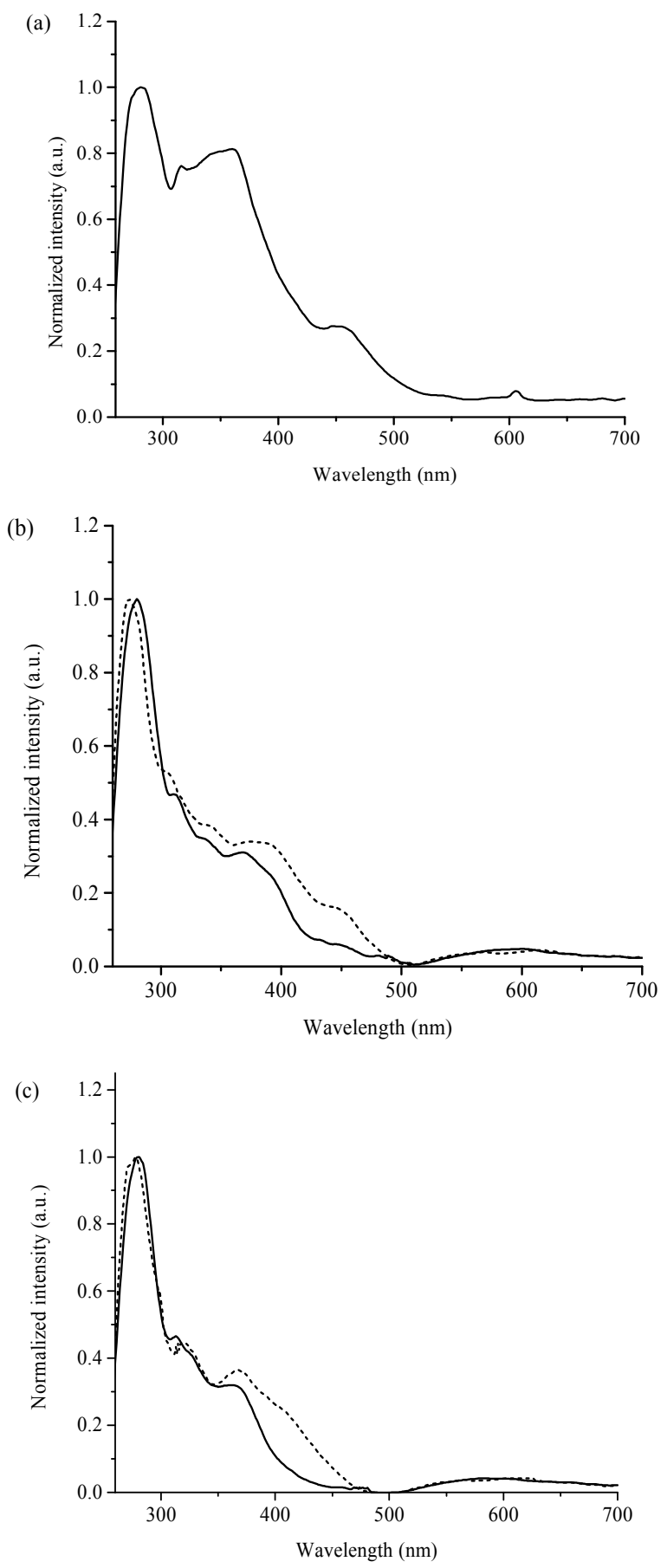

Figure S2. Excitation spectra of (a) 11 (plain curve, emission at $602 \mathrm{~nm}$ ); (b) 14 (plain curve, emission at $496 \mathrm{~nm}$; dashed curve, emission at $617 \mathrm{~nm}$ ) and (c) 19 (plain curve, emission at $474 \mathrm{~nm}$; dashed curve, emission at $613 \mathrm{~nm}$ ) in degassed DMSO at $298 \mathrm{~K}$. 

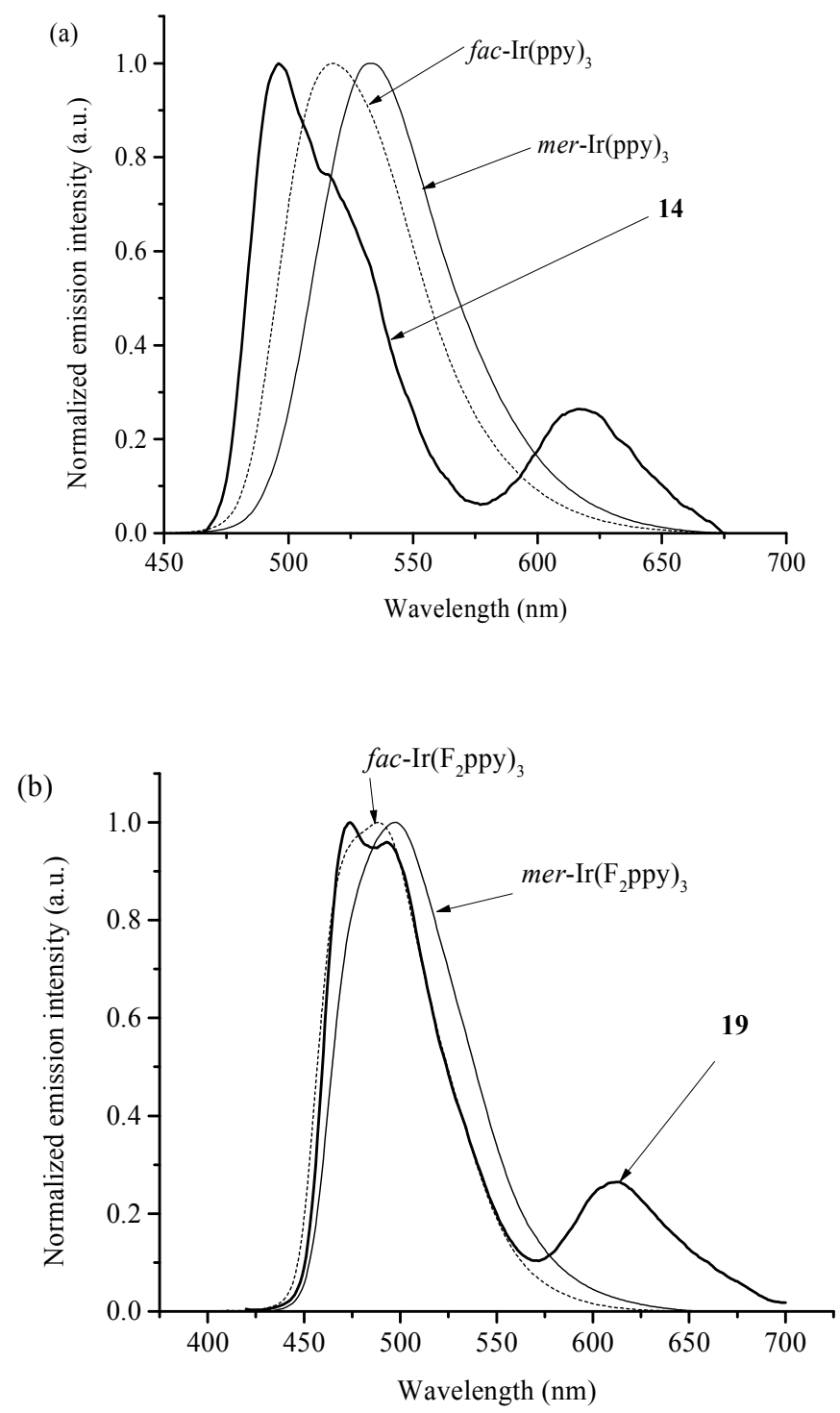

Figure S3. Emission spectra of (a) 14 with its $f a c$ - and mer-Ir(ppy) $)_{3}$ complexes and (b) 19 with its $f a c$ - and mer-Ir( $\mathrm{F}_{2}$ ppy $)_{3}$ complexes in degassed DMSO at $298 \mathrm{~K}$ (excitation at 366 $\mathrm{nm})$. 


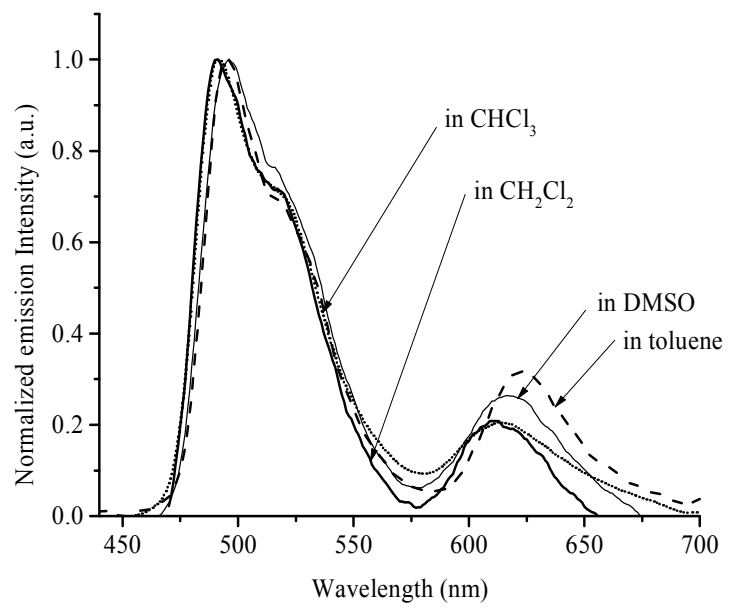

Figure S4. Emission spectra of $\mathbf{1 4}(10 \mu \mathrm{M})$ in $\mathrm{CHCl}_{3}$ (short dotted curve), $\mathrm{CH}_{2} \mathrm{Cl}_{2}$ (short bold curve), DMSO (plain curve) and toluene (dashed curve) at $298 \mathrm{~K}$ (excitation at $366 \mathrm{~nm}$ ).

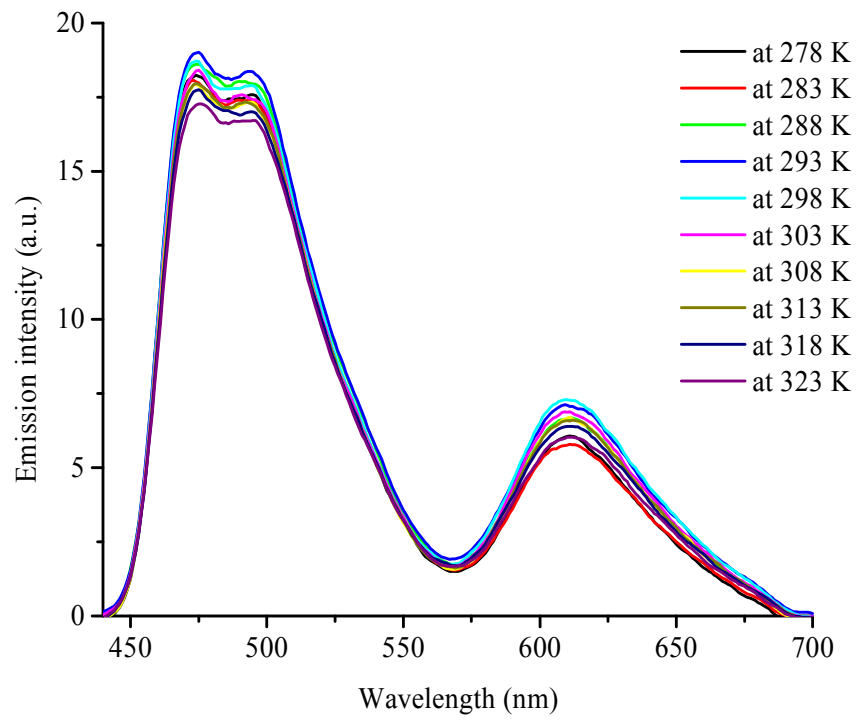

Figure S5. Emission spectra of 19 in degassed DMSO at $278 \mathrm{~K}$ to $323 \mathrm{~K}([\mathbf{1 9}]=10 \mu \mathrm{M}$ and excitation at $366 \mathrm{~nm}$ ). 


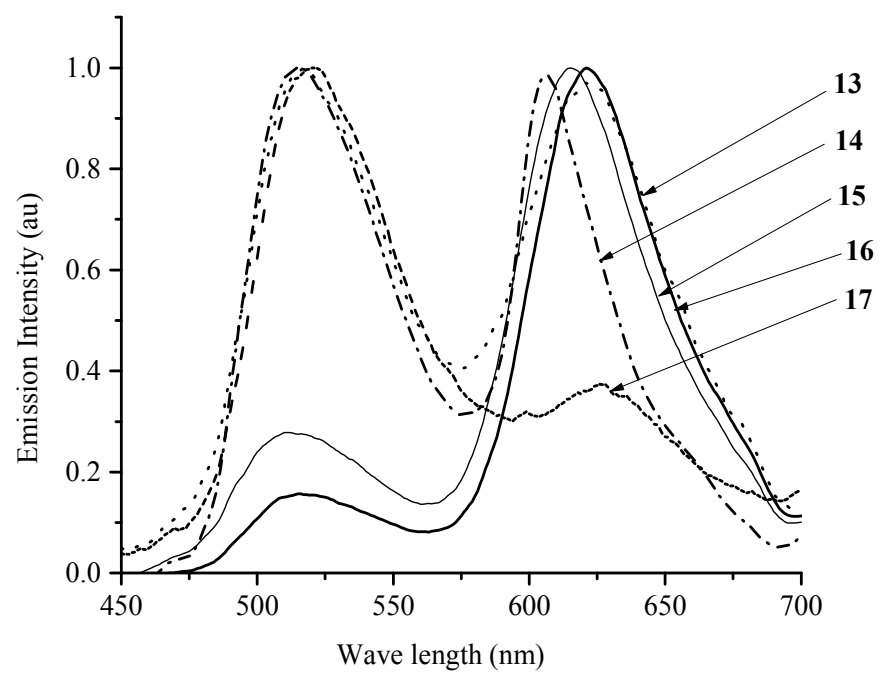

Figure S6. Solid state emission spectra of 13 (dotted curve), 14 (dashed dot), 15 (plain curve), 16 (bold curve) and 17 (short dotted curve) at $298 \mathrm{~K}$ (excitation at $366 \mathrm{~nm}$ ).

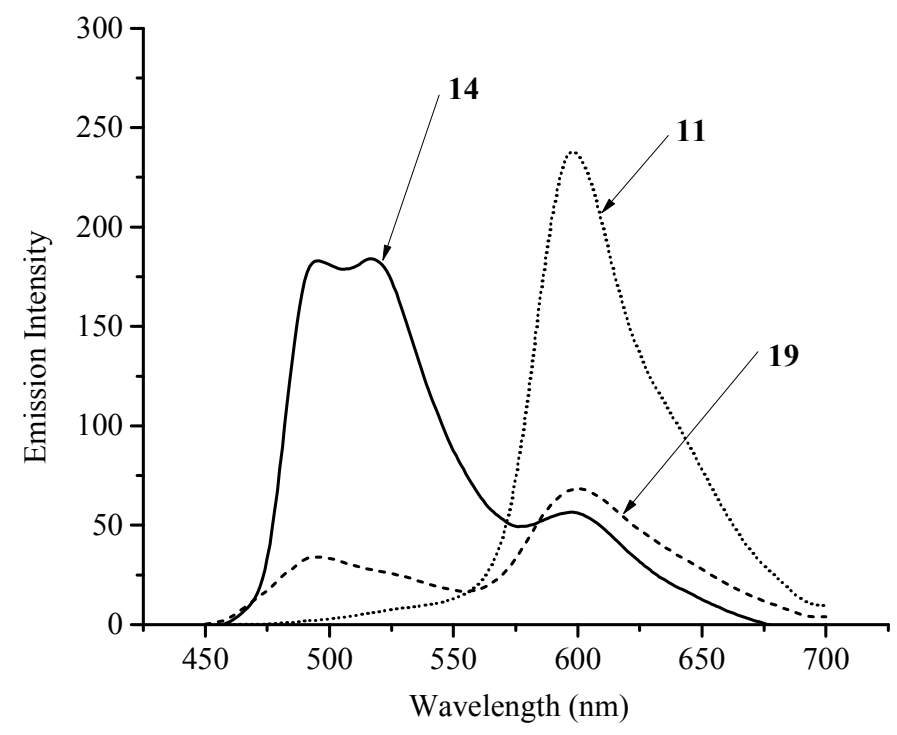

Figure S7. Emission spectra of 11 (short dotted curve), 14 (bold curve) and 19 (dashed curve) in $5 \mathrm{wt} \%$ doped PMMA film (excitation at $366 \mathrm{~nm}$ ) 

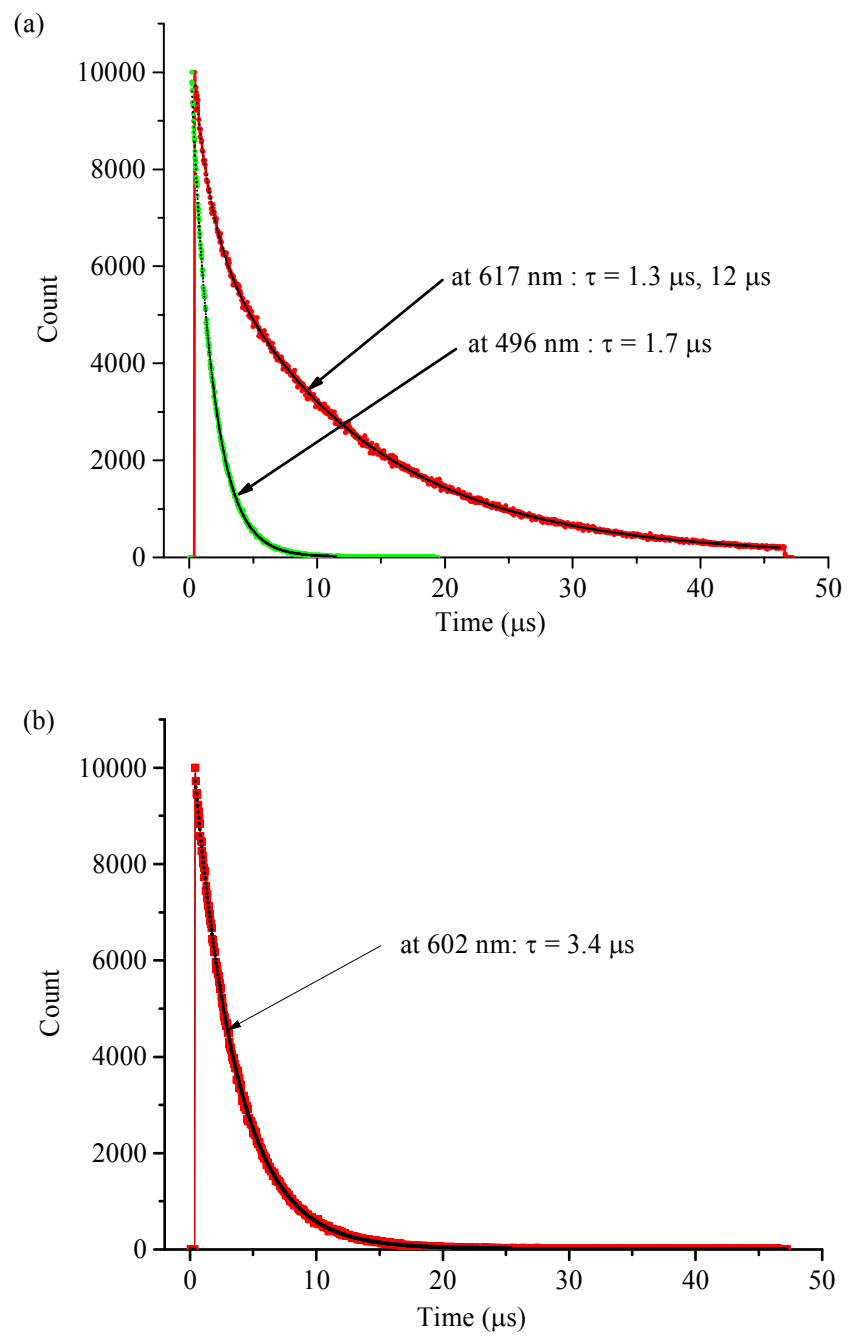

Figure S8. Emission decay graphs at emission wavelength of (a) 14 and (b) $11(10 \mu \mathrm{M})$ in degassed DMSO at $298 \mathrm{~K}$ (excitation at $371 \mathrm{~nm}$ ). Black curve lines are good fitted exponential curves. 
Table S1. RBG and xy color coordinates of 11, 14, 19 and 20 in comparison with those of 37 , $1 \mathrm{a}$ and 2.

\begin{tabular}{ccccccc}
\hline Compound & Temperature & \multicolumn{3}{c}{ RGB value } & & $x y$ color coordinate \\
& & Red & Green & Blue & $x$, & $y$ \\
\hline fac-Ir( $\mathrm{SO}_{2}$ PhClmppy $)_{3}, \mathbf{3 7}$ & $298 \mathrm{~K}$ & 27 & 249 & 247 & 0.22, & 0.33 \\
\hline$f a c-\operatorname{Ir}(\mathrm{tppy})_{3}, \mathbf{1 a}$ & $298 \mathrm{~K}$ & 51 & 240 & 41 & 0.30, & 0.58 \\
& & & & & & \\
\hline$f a c-\operatorname{Ir}(\mathrm{mpiq})_{3}, \mathbf{2}$ & $298 \mathrm{~K}$ & 242 & 48 & 41 & 0.60, & 0.33 \\
\hline $\mathbf{1 1}$ & $298 \mathrm{~K}$ & 254 & 95 & 68 & 0.54, & 0.35 \\
& $77 \mathrm{~K}$ & 254 & 85 & 89 & 0.52, & 0.32 \\
\hline $\mathbf{1 4}$ & $298 \mathrm{~K}$ & 252 & 249 & 194 & 0.34, & 0.38 \\
& $77 \mathrm{~K}$ & 254 & 232 & 190 & 0.35, & 0.37 \\
\hline $\mathbf{1 9}$ & $298 \mathrm{~K}$ & 215 & 228 & 191 & 0.32, & 0.37 \\
& $77 \mathrm{~K}$ & 253 & 253 & 253 & 0.31, & 0.32 \\
\hline $\mathbf{2 0}$ & $298 \mathrm{~K}$ & 226 & 234 & 205 & 0.32, & 0.36 \\
& $77 \mathrm{~K}$ & 253 & 253 & 254 & 0.31, & 0.32 \\
\hline
\end{tabular}

